\title{
Germanica
}

\section{«Ce qui arriva après que Nora eut quitté son mari ou les soutiens des sociétés » (1977). Une récriture d'Ibsen par Elfriede Jelinek}

Was geschah, nachden Nora ihren Mann verlassen hatte oder Stützen

der Gesellschaften (1977). Eine Umarbeitung von Elfriede Jelinek

\section{Christian Klein}

\section{(2) OpenEdition}

\section{Journals}

Édition électronique

URL : http://journals.openedition.org/germanica/2075

DOI : 10.4000/germanica.2075

ISSN : 2107-0784

Éditeur

Université de Lille

\section{Édition imprimée}

Date de publication : 31 décembre 2002

Pagination : 129-142

ISBN : 9782913857100

ISSN : 0984-2632

\section{Référence électronique}

Christian Klein, « «Ce qui arriva après que Nora eut quitté son mari ou les soutiens des sociétés » (1977). Une récriture d'Ibsen par Elfriede Jelinek », Germanica [En ligne], 31 | 2002, mis en ligne le 19 mars 2013, consulté le 06 octobre 2020. URL : http://journals.openedition.org/germanica/2075 ; DOI : https://doi.org/10.4000/germanica.2075

Ce document a été généré automatiquement le 6 octobre 2020.

(c) Tous droits réservés 


\title{
«Ce qui arriva après que Nora eut quitté son mari ou les soutiens des sociétés » (1977). Une récriture d'Ibsen par Elfriede Jelinek
}

\author{
Was geschah, nachden Nora ihren Mann verlassen hatte oder Stützen \\ der Gesellschaften (1977). Eine Umarbeitung von Elfriede Jelinek
}

Christian Klein

I

En 1977 l'auteure autrichienne Elfriede Jelinek publie sa première pièce de théâtre « Ce qui arriva après que Nora eut quitté son mari ou les soutiens des sociétés $»^{1}$. Le titre réunit une référence affichée à deux pièces de l'auteur dramatique norvégien Henrik Ibsen (1828-1906) : Maison de Poupée (1879) et Les Soutiens de la société (1877). On notera que le titre se donne d'abord à lire comme un programme picaresque qui annonce un nouvel épisode des « aventures » de Nora Helmer, à l'ombre de l'autorité d'Ibsen, mais que la conjonction clôt aussitôt cette attente en imposant un second intertexte. Le lecteur averti remarquera que le second titre subit une modification dialogique : Stützen der Gesellschaft devient Stützen der Gesellschaften. Le pluriel revient sur la polysémie du terme initial: en effet, dans la pièce d'Ibsen, "la société " désigne tantôt la communauté «dans » laquelle les personnages vivent, dont ils acceptent ou subissent les normes, « au nom de laquelle » chacun prétend parler et agir, tantôt le mot désigne «la » société, c'est-à-dire l'entreprise dans laquelle et pour laquelle on travaille, ici la compagnie maritime du consul Bernick ${ }^{2}$. L'introduction du pluriel lève l'ambiguïté qui nourrit les dialogues ibséniens et met en scène l'hypocrisie de l'armateur qui dissimule ses intérêts privés derrière l'intérêt collectif et affiche une irréprochabilité de façade. Le pluriel, à y regarder de près, indique que le propos de Jelinek n'est pas de dénoncer une nouvelle fois les sordides manigances d'un « respectable» bourgeois. Ce travail a 
déjà été effectué par Ibsen. Mais il s'agit de projeter le monde de l'économie, celui des grandes entreprises modernes, dans l'univers de la tragédie. Dans cette perspective, Jelinek a dépouillé de nombreux magazines du monde des affaires et s'est inspirée de la biographie de Hans Schleyer, président du BDI et du BDA (Association Fédérale des Chefs d'Entreprise) pour écrire sa pièce ${ }^{3}$. La suppression de l'article défini investit les grandes firmes qui contrôlent l'économie moderne, avec leurs ramifications occultes et leurs intrigues, comme un personnage. L'analyse du titre nous met déjà en garde, nous y reviendrons, contre une lecture unilatéralement socio-économique de la pièce.

2 On connaît le propos de la pièce d'Ibsen : Nora, mariée à l'avocat Torwald Helmer et mère de trois enfants, mène une existence modeste et enjouée de femme d'intérieur. Le couple s'apprête à fêter Noël en famille et aussi la récente nomination de Torwald à la direction de la banque, ce qui représente la fin des restrictions. Autrefois, Nora a contracté secrètement un emprunt et signé un faux pour emmener l'homme qu'elle aime, gravement malade, en Italie, où il guérit. Depuis elle rembourse en cachette à l'insu de son mari - un homme qui ne transige pas sur les principes - auprès d'un ancien avocat, Krogstad, convaincu autrefois de malversations. Menacé de perdre son emploi à la banque, ce dernier exerce un chantage sur Nora, qui refuse de se confier à son mari, de peur de le blesser dans sa fierté et de heurter ses principes. Le mari apprend la vérité et réagit violemment. Il s'agit avant tout pour lui de préserver son honneur. En fin de compte, le méchant renonce à son projet et le mari " pardonne » à son épouse. C'est alors que celle-ci, à la suite d'une longue explication, rompt avec lui et quitte le foyer conjugal, laissant un vide sur la scène :

Nora: Tu ne m'as jamais comprise... On m'a fait grand tort. D'abord papa, puis toi [...] Vous ne m'avez jamais aimée [...] Quand j'étais chez papa [...] il m'appelait sa petite poupée et il jouait avec moi comme je jouais avec mes poupées. Et puis je suis venue dans ta maison [...] Je veux dire que je suis passée des mains de papa dans les tiennes [...] Notre foyer n'a jamais été rien d'autre qu'une salle de récréation. Ici, j'ai été ton épouse-poupée, tout comme à la maison, j'étais l'enfant-poupée de papa. Et mes enfants, à leur tour, ont été mes poupées ${ }^{4}$.

3 Femme-objet, elle décide de quitter mari et enfants pour se chercher et se réaliser pleinement :

Nora : il faut que je veille à m'éduquer moi-même. Et cela, tu n'es pas homme à m'y aider. Il faut que je sois seule pour le faire ${ }^{5}$.

4 Jusque-là, la pièce est construite dans la tradition du mélodrame, avec ses rebondissements, les adjuvants incertains, le méchant qui se ravise, le mari attentionné mais tyrannique etc. Mais le dialogue qui réunit et sépare à la fois les deux époux fait basculer le mélodrame dans la tragédie moderne. La décision de Nora oblige à réexaminer l'ensemble du personnage, à découvrir derrière l'ingénue une femme plus complexe, contradictoire, tout à la fois « spontanée » et dissimulatrice. On devient plus attentif au langage d'Helmer, et on découvre qu'il s'adresse à sa femme presque toujours à la troisième personne - le tutoiement direct est exceptionnel avant le règlement de compte final. Le langage sert plus souvent d'écran que de moyen d'expression. Les personnages font preuve d'une certaine inaptitude à la communication.

5 Le projet de Jelinek d'imaginer et de proposer une "suite " à Une Maison de Poupée d'Ibsen s'inscrit au carrefour de deux lignes de force, l'une qui trace la réception chaotique de la pièce norvégienne sur les scènes allemandes et autrichiennes depuis 1881, l'autre, plus récente, témoigne de son appropriation explicite par le mouvement 
de libération des femmes, tant aux États-Unis, qu'en France et dans les pays de langue allemande.

6 La réception de Une Maison de Poupée sur les scènes allemandes est problématique dès sa création le 21 novembre 1880 au Residenztheater de Berlin. Ibsen doit écrire une autre fin, qui réconcilie les époux Helmer, sur pression conjointe du directeur du théâtre Thalia de Hambourg, de l'actrice Hedwig Niemann-Raabe ${ }^{6}$ et du traducteur. Les noms sont germanisés. La représentation n'est pas un succès. Le public manifeste son impatience et sa réprobation à coup de sifflets intempestifs. Le critique Paul Lindau partage l'incompréhension des spectateurs et s'appuie sur les valeurs du système patriarcal : Nora a une attitude puérile. Son rôle d'épouse est de soutenir son mari, de lui insuffler la force d'affronter «le dur combat de la vie». Elle doit se montrer une conjointe tendre, gaie, compréhensive et être une mère aimante. La résistance du public contemporain se concentre sur le troisième acte, lorsque Nora prend conscience que le «prodige » n'a pas et n'aura pas lieu et prend congé de sa prison dorée : les contemporains refusent ce qu'ils interprètent comme une rupture dans le jeu du personnage, une métamorphose inattendue. Enfin, le critique craint que cette « victoire des idées malsaines " sur la raison ne tourne la tête à des "organismes féminins " fragiles ${ }^{7}$.

7 Le public viennois manifeste le même agacement. Si Ludwig Held reproche à la pièce une absence de motivations convaincantes, et des faiblesses psychologiques $^{8}$, en revanche Max Bernstein salue, en 1887, la rigueur de la progression dramatique, la profondeur de l'analyse psychologique, la finesse des caractères. Cet hommage appuyé au dramaturge ne l'empêche cependant pas de condamner, avec le public viennois, l'attitude de Nora sur un point: on peut pardonner à la femme, face à l'égoïsme du mari, mais " pas à la mère qui abandonne ses enfants innocents »'.

8 Hermann Bahr, qui pourfend publiquement le naturalisme (1891), tente de soustraire Ibsen au "réalisme social ", Alfred Kerr veut voir en lui une synthèse avec le symbolisme. Mais aucun ne s'intéresse à Une Maison de poupée ${ }^{10}$.

9 Dans son introduction à la traduction des œuvres complètes d'Ibsen, en 1900, le directeur du Burgtheater Paul Schlenther dénie toute exemplarité à Nora :

Sie ist ein Wesen für sich [...] Nora ist kein Typus mehr. Zahllose Frauen machen in der Ehe früher oder später Noras Erfahrungen, aber sie folgen nicht Noras Beispielen. Sie halten aus und halten Haus dem «fremden Mann » [...] Sie ist eine Ausnahme und möge es bleiben ${ }^{11}$.

Le jeu de mot ([H]aus-halten : tenir une maison/supporter une situation) entend, par une pseudo-équivalence homophonique, maintenir la femme près de ses fourneaux et la condamner à la résignation.

11 Le syndrome de Médée persistera encore pendant plusieurs décennies. Il faudra le talent de la grande comédienne italienne Eleonora Duse pour imposer une vision positive du personnage-titre plus conforme aux intentions d'Ibsen au cours de tournées qu'elle effectue en Autriche et en Allemagne entre 1892 et 1909. Un critique salue son interprétation:

Eben noch ein Kind [...] wird Nora zum Charakter, der sein Recht vertritt. Der Dialog mit dem Gatten ist ein Meisterstück. Jedes Wort quillt aus der Tiefe eines kochenden Herzens, der ganze Mensch spricht, ein ganzes Menschenleben plädiert in ungewollter Beredsamkeit für seine Geltung ${ }^{12}$. 
12 Boudée par les expressionnistes, l'œuvre d'Ibsen participera à la «renaissance nordique » du III ${ }^{e}$ Reich, qui saluera les pièces à caractère historique d'avant 1875 , comme Guerriers à Helgeland (1858), inspirée de sagas islandaises, ou Les Prétendants à la Couronne (1863), mais refusera les drames sur des sujets contemporains comme Hedda Gabler ou Une maison de Poupée $e^{13}$.

13 Après la guerre, les théâtres répugnent à monter des textes auxquels on reproche encore un "déterminisme total ${ }^{14}$. En 1971 la mise en scène remarquée de Peer Gynt à la Schaubühne de Berlin, inaugure une renaissance d'Ibsen sur les scènes allemandes et autrichiennes. Tout au long des années soixante-dix, on monte plusieurs pièces par an : Les Revenants, Le Canard sauvage, Hedda Gabler. Une Maison de poupée est jouée successivement à Stuttgart (1972), Oldenburg (1973/74), Bielefeld, Freiburg, Lucerne, Berlin (1975/76), Hambourg, Düsseldorf, Bremerhaven (1977). Cet engouement culmine avec la célébration du $150^{\mathrm{e}}$ anniversaire de la naissance d'Ibsen en $1978^{15}$. Parmi ces nombreuses mises en scène, nous retiendrons celles de Neuenfels à Stuttgart (1972) et de Noelte à Berlin (1975/76) qui réactualisent la lecture de la pièce et cadrent sa réception.

14 À la différence de son auteur, qui prenait ses personnages sous sa protection, Neuenfels impose une distance critique tant par rapport à Nora qu'à son mari. Chacun est exposé, disséqué et mis en scène comme des constructions dramatiques, "des insectes dressés » note un critique ${ }^{16}$. Nora, interprétée par Elisabeth Trissenaar, joue tour à tour l'humilité de l'animal domestique, la soumission et, dès qu'elle en a l'occasion, humilie à son tour son amie d'enfance, $\mathrm{M}^{\text {me }}$ Linde, qui revient d'un exil en Amérique où elle connut de dures années de misère. Elle lui fait le récit de son "exploit» en s'attendrissant sur elle-même et dans l'espoir visible de s'assurer la reconnaissance éternelle et la protection matérielle de son mari. Peter Roggisch, dans le rôle du mari, promène avec suffisance des airs de dompteur imbu de son bon droit. Le départ de Nora n'est pas une libération, mais une fuite. Elle ne sort pas par la porte, mais enjambe une fenêtre de plain-pied en s'aidant d'une échelle apportée sur son ordre, et, une fois dehors, revient sur ses pas et jette encore un regard à travers la vitre vers l'intérieur : hésitation, remords?

En décembre 1976, Rudolf Noelte propose une lecture intimiste. Cordula Trantow est une Nora active, trop occupée pour penser à soi, pleine d'un charme enfantin. Certes, elle prend conscience de sa situation au cours de l'acte III et prend de l'ascendant sur son mari. Mais l'égoïsme de celui-ci est fortement tempéré. Il rentre de la soirée chez les voisins un peu éméché, ce qui lui assure une certaine sympathie chez les spectateurs. Et surtout, Noelte procède à des coupures significatives. Ainsi, Nora ne réclame plus son alliance, Helmer ne propose plus de lui écrire, ou de lui envoyer des colis etc. et ne se heurte plus à un triple non catégorique. Est supprimé le passage sur la religion, ce "guide infaillible» mis en avant par Torwald comme autorité de substitution et de secours sur sa femme. Cette coupure entraîne aussi l'élision de la réponse de Nora :

Hélas, Torwald, le fait est que je ne sais pas au juste ce qu'est la religion [...] Quand j'aurai quitté tout ça ici et que je serai seule, j'examinerai cette question comme les autres. (Une Maison de poupée... 218)

Le texte allemand prend un sens programmatique plus net encore :

Wenn ich aus alledem heraus bin und allein dastehe, will ich auch der Sache auf den Grund gehen. (Reclam 90) 

l'impasse sur les causes profondes de la crise. Le spectacle a perdu sa dimension revendicative et rebelle initiale. C'est un plaidoyer, très applaudi notons-le, qui s'intéresse à la représentation des souffrances d'un couple en crise, loin des fanfares sur l'émancipation de la femme ${ }^{17}$. $\mathrm{du}$ XIX $\mathrm{X}^{\mathrm{e}}$ siècle le mouvement cite la pièce d'Ibsen pour dénoncer la dégradation de la condition féminine dans le mariage ${ }^{18}$. Dans son texte fondateur de 1949 , Simone de Beauvoir évoque l'héroïne d'Ibsen qui a accepté «bon gré mal gré » pendant huit ans que «l'homme pense à sa place », mais qui « comprend » que le mariage doit être «la mise en commun de deux existences autonomes, non une retraite, une annexion, une fuite, un remède ». Elle ajoute :

C'est ce que comprend Nora quand elle décide qu'avant de pouvoir être une épouse et une mère, il lui faut devenir d'abord une personne ${ }^{19}$

Betty Friedan, pionnière du féminisme américain, rend aussi hommage à la révolte de Nora :

Ibsen a montré qu'il comprenait ce qu'était le féminisme en écrivant, en 1879, « [Une] Maison de poupée » où il démontrait symboliquement qu'une femme était tout simplement un être humain [...] Des milliers de femmes de classe moyenne, en Europe aussi bien qu'en Amérique, se reconnurent en Nora et en 1960, près d'un siècle plus tard, des millions de ménagères américaines qui suivirent la pièce à la télévision se reconnurent aussi $[. .].]^{20}$.

On retrouve cette référence dans l'ouvrage théorique du Women's Liberation Movement, Sexual Politics de Kate Millett (1969), qui écrit en particulier :

La Nora Helmer d'Ibsen est la véritable instigatrice de la révolution sexuelle [...] Maison de poupée et Salomé [d'Oscar Wilde] sont des drames de l'affrontement [...] Nora devait affronter tous les conformismes et les préjugés chevaleresques masculins, qui la réduisaient à la dimension d'un jouet d'enfant, dans l'espoir qu'elle resterait éternellement ce petit être, cet animal familier de la maison [...] Lorsque Wilde eut échoué en 1895, il restait à Nora et à sa bande de révolutionnaires quelques bonnes années d'insurrection devant elles ${ }^{21}$.

21 Tous ces ouvrages ont été traduits en allemands et sont lus par l'ensemble des féministes, y compris par Elfriede Jelinek. En 1975, Alice Schwarzer publie Der «kleine Unterschied " und seine großen Folgen ( $L a$ "petite différence » et ses grandes conséquences), et fonde la revue Emma en 1977.

Le mouvement féministe des années soixante-dix en Allemagne lutte contre le $\$ 218$ et pour le droit à l'avortement, il veut une sexualité libérée de la domination masculine, ce qui conduit, dans ses formes radicales, à prendre de plus en plus ouvertement position contre le mariage et les enfants. Il dénonce le travail non-rétribué de la femme au foyer comme une forme moderne d'esclavagisme légalisé.

23 Elfriede Jelinek se situe à la confluence de ces deux réceptions, théâtrale et féministe. La première est fortement contrastée et oscille entre une lecture combative et une lecture intégrative, une esthétique qui souligne les fractures et les meurtrissures et une autre qui décontextualise les conflits. La seconde transforme Nora en un mythe révolutionnaire. Il en ressort que, dans son travail de réécriture, Jelinek s'intéressera moins au texte source pour tenter d'en capter des significations cachées que pour mettre en perspective la réception controversée d'un mythe. 
La pièce d'Ibsen a une structure pyramidale classique en trois actes, de forme fermée (Klotz), où chaque élément dramatique est centré autour d'un conflit principal, avec l'introduction d'événements anciens dont la révélation progressive nourrit la progression de l'intrigue. La fin ouverte, cependant, rompt avec la dramaturgie initiale. Helmer se retrouve seul sur scène. Le spectateur s'interroge: que deviendra Nora? Ibsen se plaisait à décliner la question et à déclarer qu'il l'ignorait. Dans la mise en scène de Nicolas Briegel, à Hambourg en 1976, Nora quitte la maison sous la pluie et s'enfonce dans un brouillard épais. Son avenir sera difficile.

Jelinek abandonne la division en actes et propose le parcours en 18 scènes ou étapes d'une passion areligieuse et désillusionnée de Nora Helmer. L'action est transposée dans l'Allemagne des années vingt, qui conduisent à la montée du fascisme, comme en témoignent des citations de Mussolini et de Hitler. La forme ouverte permet de casser la logique du drame naturaliste et d'afficher l'intertextualité comme principe d'écriture. En effet, les personnages avouent volontiers leur origine littéraire :

Nora : Ich bin Nora aus dem gleichnamigen Stück von Ibsen (9)

Ou encore :

Weygang : Wie heißt du?

Nora : Nora.

Weygang: Wie die Hauptfigur des Theaterstücks von Ibsen?

Nora: Was Sie alles wissen! (25)

$\mathrm{Ou}$ :

Minister: Sie hat eine starke Kindlichkeit wie beispielsweise Wedekinds Lulu sie auch hat. (33)

et se présentent comme des constructions dramatiques qui favorisent les déformations signifiantes. Jelinek justifie ce procédé de la distorsion par les contraintes du genre dramatique :

Wenn ich Theaterstücke schreibe, dann bemühe ich mich nicht, psychologisch agierende Personen auf die Bühne zu stellen. Das soll, meine ich, dem Film vorbehalten bleiben. Ich vergrößere (oder reduziere) meine Figuren ins Übermenschliche, ich mache also Popanzen aus ihnen, sie müssen ja auf einer Art Podest bestehen. Die Absurdität der theatralischen Situation [...] verlangt eben diese Übersteigerung der Personen ${ }^{22}$.

Nora est embauchée dans une usine où elle est remarquée par l'industriel Weygang alors qu'elle répète la tarentelle pour une fête du comité d'entreprise. Il l'installe chez lui et l'engage comme espionne auprès du directeur de la banque Conti, son ex-mari Helmer, qu'elle fouette au cours de séances sado-maso. Weygang la "cède » à un ministre et la renvoie quand sa beauté physique manifeste les premiers signes de dégradation. Elle retourne chez Helmer, meurtrie, rêvant encore d'un amour romantique impossible avec son chevalier d'industrie. Dans la scène finale, la radio annonce une nouvelle fusion du groupe TEXO dans la chimie, l'incendie de l'usine Payer-Fasern, on entend les premiers discours et les bruits de bottes du fascisme allemand. L'antisémitisme pointe sa haine dans le foyer Helmer.

Les reprises citationnelles sont assez nombreuses pour assurer un effet de reconnaissance. Jelinek reprend une partie des personnages : outre le couple Helmer, l'avocat-maître chanteur Krogstad, l'amie d'enfance Mme Linde, la gouvernante Anne. 
Les enfants, maintenus hors champ chez Ibsen, surgissent brièvement à la fin de la scène 11. Weygang, qui " remplace » Helmer auprès de Nora, reprend à son compte le langage hypochoristique d'origine zoologique du mari («mon alouette» 38-39), la taquine dans les mêmes termes sur son caractère dépensier (ou prétendu tel, chez Ibsen ${ }^{23}$, recourt à la même métaphore sur «l'oiseau qui baisse les ailes » quand Nora boude ${ }^{24}$. Weygang, comme Helmer, a le «sang qui bout » en voyant Nora danser la tarentelle, etc. La reprise textuelle signale une reprise... en mains de Nora par la nouvelle autorité masculine. Cette fois, les marques d'affection d'Helmer, qui manifestent aussi, il est vrai, une infantilisation et une mise sous tutelle de sa partenaire, sont mises au service d'une instrumentalisation réfléchie de la part du nouveau maître. La dépendance de Nora a augmenté d'un cran. La réécriture de la scène où Nora danse la tarentelle montre l'enjeu d'une relecture.

31 La tarentelle est une danse du sud de l'Italie. Elle a, dans les pays nordiques, la réputation d'être très érotique ${ }^{25}$. Ibsen la présente sous sa forme policée. Helmer l'a apprise à Nora, qui la danse dans les soirées mondaines. Dans Une maison de poupée, Nora répète dans le salon. Elle s'y adonne « avec fougue » dans l'attente de la lettre de Krogstad qui doit tout révéler à Helmer. Ce langage non-verbal a une fonction expressive (le désespoir de Nora), mais aussi dramatique, car il s'agit de détourner l'attention du mari et de retarder l'échéance. Au delà, la scène exprime une vitalité refoulée. Dans la pièce de Jelinek, Nora répète dans le cadre d'un programme culturel de l'entreprise qui doit recevoir la visite d'un groupe industriel. Elle danse avec la même fougue. Dans les deux scènes, Nora subit l'intervention critique de l'Homme (le mari et le contremaître), et le commentaire insistant de l'auteur(e) sur la frénésie croissante qui s'empare de Nora. Les deux interventions s'opposent : nicht so stürmisch vs sie tanzt immer wilder. Ce qui exprimait le désespoir dans le texte source traduit la joie de faire vivre son corps, de participer à une fête collective. Mais le contremaître pose de nouvelles exigences. Alors que Helmer entendait maintenir les contorsions de sa femme dans les limites de la décence, le contremaître demande " plus de sensualité » et plus de «lubricité» (« etwas sinnlicher», «zu ungeil»). De sorte que l'énergie intérieure que libère la jeune femme, signe fort de sa féminité, tombe sous l'interdit masculin parce qu'il échappe à sa marchandisation. Le malaise d'Helmer - qui plaçait son orgueil bourgeois dans les prouesses contrôlées de son épouse - cède la place à un diktat autrement plus redoutable: celui de l'argent. Ainsi que le fait remarquer le nouveau commanditaire : l'entreprise paie la prestation, alors que le mari ne payait rien. Le rappel de la Loi de l'argent conforte la thèse féministe qui met en parallèle le travail non rémunéré de la ménagère et celui des femmes salariées. La reprise dénonce l'hypocrisie de la situation de la ménagère et présente le salariat comme une autre forme d'esclavage. La réplique cinglante de Nora contre toute forme de pornographie directement empruntée aux actions du MLF - est elle-même remise en question dès que Nora se jette, sans transition, dans les bras de l'industriel Weygang qui observait ses charmes depuis un moment, en abdiquant toute fierté et conscience de soi. Jouant sur les effets de reconnaissance, la reprise installe avec force l'impossibilité d'échapper au « regard masculin » et à la Loi de l'argent.

Dans le même temps, le personnage du mari subit une démultiplication : Nora refuse les avances d'un contremaître prétentieux et tyrannique, qu'elle juge trop petit-bourgeois, tombe amoureuse d'un homme d'affaires sans scrupules, joue la domina dans les hautes sphères de la politique et de la finance, pour se retrouver chez le mari qu'elle a 
contribué à ruiner. Toutes ces figures masculines incarnent avec cynisme les valeurs du système patriarcal :

Weygang : Der Mann hat eine Begierde und einen Trieb. Die Frau ist Gegenstand des Triebes (29).

Selon lui, le mâle triomphe par la destruction, le combat et la violence. La beauté de la femme est une marchandise périssable (31), etc. Ce langage cru, aux accents nietzschéens, contribue à une typisation des partenaires masculins qui confine à la caricature. La diffraction du "héros» masculin permet d'installer une galerie d'individus fortement hiérarchisés entre eux, où chacun copie l'autre, mais à un niveau social toujours inférieur. C'est ainsi que Helmer, qui entretient une liaison perverse avec Mme Linde, l'ancienne amie d'enfance de Nora, se vante auprès du puissant Weygang d'"être né pour spéculer sur des faits et des chiffres", il se montre particulièrement obséquieux et servile. À son tour, il « cède " $\mathrm{M}^{\mathrm{me}}$ Linde à Krogstad. Chaque "redistribution" des "rôles " féminins par un mâle bivalent - servile envers les puissants et brutal envers la femme considérée comme un être inférieur commente la précédente avec une perte en précaution oratoire, une déperdition de vernis social et un dépouillement moral accru. On peut être tenté de commenter cette typisation comme une illustration des thèses féministes les plus radicales. Il est vrai que les prises de position d'Elfriede Jelinek soutiennent ces thèses ${ }^{26}$. Dans sa thèse Sabine Perthold s'appuie sur les grands textes du marxisme pour analyser la condition féminine depuis le Moyen Âge, l'esclavage moderne de la femme, par exemple ce discours de Bebel en 1890 à Nuremberg :

Wie der Arbeiter vom Kapitalismus unterjocht wird, so die Frau vom Manne, und sie wird unterjocht bleiben, solange sie nicht wirtschaftlich unabhängig ist ${ }^{27}$.

Perthold cite des études sociologiques récentes qui confirment que le travail ne libère pas la femme ${ }^{28}$. Alice Schwarzer associe l'ouvrière et la femme au foyer :

Frauen sind Sklavinnen im Haus, wo die männerdominierte Berufswelt nicht mehr ist als ein Schauplatzwechsel. Frauen bleiben auch im Beruf die machtlosen Exekutantinnen der Männer ${ }^{29}$.

Pour autant, cette lecture sociologique de la pièce tend à réduire le texte de Jelinek à une illustration de thèses défendues depuis longtemps par simone de Beauvoir, reprises et complétées au cours des années soixante-dix par Susan Sontag, Alice Schwarzer, Luce Irrigaray etc., et qui sont certes partagées par Jelinek, mais qui ne rendent pas compte de la complexité de son écriture. L'industriel Weygang, par exemple, est sexuellement attiré par Nora, il la désire fortement. Son langage est d'abord celui du mâle habitué à conquérir et à s'approprier l'énergie de la femme, comme source de régénération. Mais le discours dérape, il s'étonne de découvrir en lui « des sentiments [qu'il] pensai[t] enfouis « et engage un duo amoureux avec elle :

Weygang: Mein Gott, was für ein beachtlicher Körper! Gäbe es solche Körper in unserm Leben nicht, nie könnten wir uns regenerieren. [...] leise : Was dem einen zu wild oder zu schnell ist, ist genau richtig für mich. Wovor eine kleinere und feigere Natur zurückschreckt, das zieht mich magisch an [...] Mir ist ja, als ob der Blitz in mich einschlägt. Wie kommt das ? [...] Plötzlich fährt etwas wie ein Pfeil durch mich hindurch [...] Was entsteht plötzlich in mir? Es wird doch nicht etwas wie ein Gefühl sein, was ich längst verschüttet glaubte ? (24 et suiv.) $)^{30}$

Certes, les deux personnages jouent une nouvelle version de la scène du coup de foudre. La naïveté des réflexions de Weygang, l'insertion d'éléments étrangers au langage amoureux (par exemple la comparaison entre la flèche de Cupidon et la blessure 
infligée par ce honteux accord de Versailles...), signalent une parodie volontairement mal ajustée du roman-photo. La complicité spontanée de Nora, en rupture avec ses allures précédentes de passionaria du féminisme, surdéterminent le caractère inauthentique de la scène. Au cours d'un entretien privé avec un chef d'entreprise ou avec le ministre, il égrène un certain nombre de lieux communs machistes comme par exemple :

Im unverdorbenen Weibe soll angeblich kein Geschlechtstrieb hausen. Nur die Liebe wohnt darin. Das Weib hat den Naturtrieb, einen Mann zu befriedigen. (30)

Mais dans le même temps, il affirme que Nora est «son rayon de soleil et son bien le plus précieux ». L'idée de se séparer d'elle «l'atteint comme un coup de poignard dans la poitrine », il l'aime et il est « devenu son esclave » (32-33). Au même ministre, qui lui confie combien « sa Nora » l'attire, il répond:

Die Frau, die ich liebe, werde ich niemals verschachern, eher verschachere ich mich selber oder meinen rechten Arm [...] Mit Nora werde ich noch im hohen Alter zusammensein, Philemon und Baucis.

Puis, il se ravise aussitôt et lui promet sa femme dès que son « extrême passion » se sera éteinte, disons... dans trois semaines (!) (34). La désinvolture de ces propos s'accorde mal avec l'amour de Philémon pour Baucis. Néanmoins cet élan de fidélité, même contredit au cours du même entretien par un revirement brutal, brouille quelque peu le message car il n'obéit à aucune nécessité dramatique. Dans la mesure où l'industriel juxtapose, puis hiérarchise, la beauté féminine et celle... du Capital, seule à rester inaltérable, on peut en conclure que cette incohérence dans l'attitude traduit la manifestation du désir (ici masculin) et sa soumission à la loi du Capital, jusque et y compris chez les dirigeants les plus avisés dudit Capital. Cette réflexion rejoint la vision d'une ouvrière, porte-parole du courant révolutionnaire hérité de Bebel et de Clara Zetkin, qui rêve d'un monde prochain « où l'être humain pourra montrer de nouveau des sentiments» (15). Les personnages masculins sont donc eux aussi prisonniers du système, même s'ils en tirent sans vergogne de nombreux avantages. Le gestus brechtien met en scène les représentants du patriarcat en leur faisant prononcer des lieux communs, un galimatias où se mêlent des considérations sur le capital, sur la nature des femmes et des ébauches de désir qui tournent au fantasme. Tels des insectes, ils s'agitent, se piétinent, sombrent ou triomphent sans répit. Ils n'ont, de par le choix affiché de Jelinek, pas d'existence propre. Ils sont la rencontre malhabile entre des êtres qui n'arrivent pas à devenir de chair et des pantins de démonstration.

On peut s'interroger sur la finalité d'une dramaturgie qui emprunte au modèle brechtien le décalage entre le dire et le faire, entre jouer et montrer pour mettre en scène une aliénation généralisée.

La référence récurrente au "sentiment » et à la «nature » des choses font apparaître un travail de déconstruction qui sous-tend l'ensemble du texte. En effet, les personnages tentent à plusieurs reprises de justifier leur attitude en invoquant un éternel féminin, ou des constantes "naturelles ». La révolutionnaire Eva, par exemple, aime sans retour le contremaître. Renonçant à toute intelligence politique, elle affirme que la femme est toujours guidée par les sentiments. Nora lui répond qu'il faut savoir oublier les sentiments (14), mais justifie sa liaison avec Weygang en déclarant que les hommes, eux aussi, « doivent avoir du temps pour les sentiments » (18). Elle qui rejette toute définition de la femme, déclare par ailleurs que les femmes sont "par nature " dotées d'un puissant sentiment de solidarité (17). La même Nora qui tient des propos 
incendiaires contre la domination masculine, cite Hitler sur « la disposition féminine du peuple dans son écrasante majorité » (16). Les représentants du monde des affaires accumulent des lieux communs sur la prétendue «nature » qui, ayant horreur du vide, aspire à sortir du chaos pour aller vers l'ordre (33). La même nature «favorise les chefs d'entreprises». Le Capital «a une nature anxieuse», il «a horreur de l'absence de profit, comme la nature a horreur du vide ».

41 Toutes ces déclarations sentencieuses relèvent tantôt d'improvisations pour justifier un avantage personnel, tantôt de clichés bêtes et ridicules. Mais elles organisent un réseau de significations, une isotopie de l'immuabilité du monde. On sait que Jelinek a lu, commenté et mis en pratique l'essai de Roland Barthes sur les mythes modernes ${ }^{31}$. Pour Barthes, le mythe « restitue une image naturelle $[\mathrm{du}]$ réel $»^{32}$. Il est « une parole volée et rendue » (211) qui « transforme l'histoire en nature » (215). Il ajoute :

En passant de l'histoire à la nature, le mythe fait une économie: il abolit la complexité des actes humains, leur donne la simplicité des essences, il supprime toute dialectique [...] (231)

À ce titre, Jelinek s'est attaqué au mythe de Nora, devenue icône du féminisme, pour inventorier la complexité de la situation féminine, en rejetant des simplifications hâtives et péremptoires. La reprise dialogique de thèmes, de personnages et de fragments de discours au texte source d'Ibsen met en évidence une stéréotypisation de mythèmes dans les discours contemporains, tant par des metteurs en scène que par des porte-parole de la libération des femmes. Par son théâtre, Jelinek entend, à l'instar de Barthes, faire œuvre de "mythologue » en portant «la destruction dans le langage collectif », en s'interdisant "d'imaginer ce que sera sensiblement le monde, lorsque l'objet immédiat de sa critique aura disparu; l'utopie lui est un luxe impossible $»^{33}$.

\section{NOTES}

1. E. Jelinek, Was geschah, nachdem Nora ihren Mann verlassen hatte oder Stützen der Gesellschaften, Vienne, Munich, Sessler, 1980. Nous citerons d'après la réédition de 1984 : Theaterstücke, éd. Par Ute Nyssen, Cologne, Rowohlt, 1984, pp. 7-78. La trad. fr. de Louis-Jacques Sirjacq, Ce qui arriva quand Nora quitta son mari, Paris, L'Arche, 1993, donne - sans le signaler - une version fortement abrégée du texte.

2. Cf. les multiples occurrences (dans les deux contextes) in : Die Stützen der Gesellschaft, trad. all. de Hans Egon Gerlach, Reclam (1960) 1998, pp. 6, 33, 39, 43, 48, 54, 56, 60, 67, 69, 71, 74, 77, 80, 85, 92, 93.

3. E. Jelinek, «Ich schlage sozusagen mit der Axt drein », in : TheaterZeitSchrift 7, 1984, p. 14.

4. Ibsen, Une maison de poupée, trad. du norvégien par Régis Boyer, Paris, Flammarion, (1988) 1994, pp. 212-214.

5. Ibid. p. 215.

6. Interrogée sur son opinion au sujet de la pièce, l'actrice s'écria : «Ich würde meine Kinder nie verlassen !!!! », Literarisches Echo 2, 1899/1900, col. 969, cit. in Aldo Keel, « Henrik Ibsen Nora (Ein Puppenheim», Erläuterungen und Dokumente, Stuttgart, Reclam, 1990, pp. 44-45 (abrév. Erläuterungen...) 
7. Paul Lindau, Nora in : Die Gegenwart, Berlin, 28/11/1880, p. 348 et suiv., cit. in Erläuterungen... 50.

8. Ludwig Held, Neuer Wiener Tageblatt, 9/9/1881, cit. in : Margot Kuhn, « Ibsens 'Nora' auf den Wiener Bühnen (1881-1971)», Maske und Kothurn 1978, p. 95.

9. Max Bernstein : « Nora » in : Münchner Neueste Nachrichten, 27/3/1887.

10. Cf. David E.R. George, Henrik Ibsen in Deutschland. Rezeption und Revision, Göttingen, Vandenhoeck \& Ruprecht, 1968, pp. 51-55.

11. Henrik Ibsens Sämtliche Werke in deutscher Sprache, durchges. u. eingel. v. Georg Brandes, Julius Elias, Paul Schlender, t. 6, Berlin, Fischer 1900, p. XXII.

12. Anonyme, Fremdenblatt, Vienne, 26/2/1992, cit. in Kuhn, op. cit., p. 102. Cf. aussi George, op. cit., p. 59: «Ihre Nora war 'passionate, sickly, neurasthenic, self-involved' [Harding, B, Age cannot wither, London, 1949, p. 72]». Elle fit forte impression sur Max Reinhardt et sur Hofmannsthal, qui lui consacra une étude («Menschen in Ibsens Dramen »).

13. "Les problèmes de Nora ne sont plus les nôtres ", écrit Otto C. A. zur Nedden, Drama und Dramaturgie im 20. Jahrhundert, Würzburg, $3^{\mathrm{e}}$ éd. 1944, p.18, cit. in : Wilhelm Friese (éd. ), Ibsen auf der deutschen Bühne, Tübingen, Niemeyer, 1976, p. XXI.

14. Joachim Kaiser, Kleines Theatertagebuch, Reinbek bei Hambourg, 1965, pp. 155-157.

15. La revue théâtrale Maske und Kothurn, publiée à Vienne, lui consacre un numéro en 1978.

16. Georg Hensel, Darmstädter Echo 5/2/1972.

17. Sur cette mise en scène, cf. les critiques de Georg Hensel, FAZ, 14/12/1976, Rolf Michaelis, Theater Heute 1977/5, pp. 6-10.

18. Cf. O. Bistram, Nora und die wahre Emanzipation der Frau, Wiesbaden, 1900, George, op. cit., p. 26. 19. Simone de Beauvoir, Le Deuxième sexe, Gallimard, coll. Folio/Essais, t. 2, p. 296 et p. 324 ; cf. trad. all., Das andere Geschlecht, rororo, p. 448, p. 696.

20. Betty Friedan, La Femme mystifiée, trad. Y. Roudy, Paris, Gonthier, (1964) 1973, p. 87 et suiv., trad. all. Der Weiblichkeitswahn, rororo 1963, p. 56 et suiv.

21. Kate Millet, La politique du mâle, trad. Elisabeth Gille, Paris, Stock, 1971, p. 178 et suiv.; trad. all., Sexus und Herrschaft, Munich, 1971, p. 179 et suiv.

22. E. Jelinek : « Ich schlage sozusagen mit der Axt drein' in : TheaterZeitSchrift 7, 1984, p. 14.

23. « Hat mein lockerer Zeisig wieder Geld verschwendet » ("Was geschah... 38), cf. : « Ist mein lockerer Zeisig wieder ausgewesen uns hat Geld verschwendet » (Ein Puppenheim, Reclam 6).

24. «Nun, nun, meine Lerche muß nicht gleich die Flügel hängen lassen » (Ein Puppenheim, Reclam 7 ; Was geschah..., 38).

25. Cf. Wilhelm Bergsoes, Gespenster-Novellen, trad. all. A. Strodtmann, Berlin, Janke, 1873, pp. 54-59.

26. Par ex. : " Unser System ist ein patriarchalisches und [die Frau] hat doch gegen zwei Gegner zu kämpfen: den phallokratischen und den kapitalistischen.», Programmheft des Schauspielhauses Graz "Was geschah...», p.11. Cf. aussi Elfriede Jelinek: "Der Krieg mit anderen Mitteln » (1984) in : Koschel/ Weidenbaum (éd. ), Kein Objektives Urteil - Nur ein lebendiges. Texte zum Werk von Ingeborg Bachmann, Piper, Munich, 1989, pp. 311 314, 316.

27. August Bebel, Die Frau und der Sozialismus. Als Beitrag zur Emanzipation unserer Gesellschaft, bearbeitet und kommentiert v. Monika Seifert, Hanovre 1974, p. 365.

28. Cf. Helge Pross, sociologue: «Frauen sind Gastarbeiterinnen in einer Männergesellschaft », cit. in: Alice Schwarzer (éd.), Lohn: Liebe. Zum Wert der Frauenarbeit, Francfort/M, 1973, p. 16. Perthold Sabine, Elfriede Jelineks dramatisches Werk. Theater jenseits konventioneller Gattungsbegriffe, Phil. Diss. Vienne, 1991.

29. Ibid., p. 16.

30. Notons que, dans la scène 14 , Krogstad reprend la même expression pour évoquer son amour d'autrefois pour Mme Linde: «Das Gefühl ist in mir verschüttet» (59). Cette circularité 
intratextuelle discrédite à rebours l'émotion de Weygang dans la scène 6 en suggérant un fonds commun de métaphores usées qui nourrit le discours masculin à travers les individus.

31. E. Jelinek : «Die endlose Unschuldigkeit » (1970) in : E.J., Die endlose Unschuldigkeit, Prosa Hörspiel - Essay, Swifting, 1980, pp. 49-82.

32. Roland Barthes, Mythologies, Seuil 1957, p. 230.

33. Ibid., p. 245.

\section{RÉSUMÉS}

La pièce d'Elfriede Jelinek (1977) Ce qui arriva après que Nora eut quitté son mari ou les soutiens des sociétés (1979) reprend et prolonge deux pièces d'Ibsen : Nora ou la Maison de poupée (1879) et Les Soutiens de la société (1877). Le présent travail étudie d'abord la réception de Nora ou la maison de Poupée sur les scènes allemandes et autrichiennes depuis sa création en particulier avec la renaissance d'Ibsen de 1972 à 1978, puis la confiscation du personnage de Nora comme exemple d'émancipation par le Women' Liberation Movement au cours des années soixante et soixantedix (S. de Beauvoir, K. Millett, A. Schwarzer) pour examiner comment le texte dramatique organise, par la réécriture du texte-source, la critique de Jelinek contre ce qui est devenu le «féminisme radical » de Nora. Jelinek s'appuie sur « Mythologie» de Barthes pour procéder à une destruction $d u$ "mythe» Nora. La pièce met en scène et dénonce les représentations stéreotypées du féminin dans leur expression contradictoire tant par les personnages féminins que masculins. L'individualisme protestataire de Nora l'isole des autres femmes et se dissout dans une alliance suicidaire avec les tenants du patriarcat. La démultiplication du « rôle » masculin montre que les représentants du capital sont à la fois les acteurs et les victimes du système.

Jelineks Stück Was geschah, nachden Nora ihren Mann verlassen hatte oder Stützen der Gesellschaften nimmt Bezug auf zwei Stücke ibsens (Nora oder das Puppenheim und Die Stützen der Gesellschaft). Die Arbeit untersucht einerseits die Rezeption von Ibsens Nora Stück auf deutschen und österreichischen Bühnen seit ihrer Uraufführung - insbesondere während der Ibsen-Renaissance von 1972 bis 1978 - und, andererseits, die Vereinnahmung von Ibsens Nora als Galionsfigur der Emanzipation durch die amerikanische und europäische Women's Liberation Movement im Laufe der sechziger und siebziger Jahre (S. de Beauvoir, B. Friedan, K. Millet, A. Schwarzer), um dann in der intertextuellen Um- und Forschreibung Jelineks Auseinandersetzung mit Noras «Radikalfeminismus » am Text nachzugehen. In Anlehnung an Barthes' "Mythen des Alltags » operiert Jelinek eine Destruktion des Mythos «Nora». Die herkömmlichen Klischees des Weiblichen werden sowohl bei den Frauen- wie bei den Männerfiguren in ihren widersprüchlichen Äußerungen entlarvend dargestellt. Noras individualistische Protesthaltung isoliert sie von den anderen Frauen und löst sich in einem selbstmörderischen Bündnis mit den Exponenten des Patriarchats auf. Die Aufspaltung der männlichen « Rolle » zeigt die Vertreter des Kapitals als Täter und Opfer des Systems.

\section{AUTEUR}

\section{CHRISTIAN KLEIN}

Université Grenoble III 